\title{
Standounkt
}

Eine Replik

\section{Zur Entwicklung des Regionalen Wohlfahrtsindex}

Landtagsinitiativen zur Umsetzung gibt es bisher nicht.

Ob die Landesregierung den einstimmigen Landtagsbeschluss überhaupt umgesetzt hat, ist nicht bekannt.

\section{Visionäre und Vordenker}

Besonders bedauere ich auch, dass in dem obigen Artikel nicht auf die Verdienste von Martin Jänicke [4] und Christian Leipert [5] hingewiesen wird, die zusammen mit anderen und mir das Institut für Ökologische Wirtschaftsforschung (IÖW) im Mai 1985 gegründet haben. Das hätte deutlich gemacht, wie lange sich das IÖW mit diesem Thema bereits befasst und welches Know-how hier inzwischen zusammengetragen werden konnte. von Rodenhä rechnung des Regionalen Wohlfahrtsindex (RWI) in Schleswig-Holstein auf eine Landtagsinitiative der SPD aus dem Jahre 1990 zurück. Rodenhäuser et al. (2017, S. 31) schrieben: „Auf der Ebene der deutschen Bundesländer wurde zunächst $2011 \mathrm{im}$ Auftrag der schleswigholsteinischen Landtagsfraktion von Bündnis 90/Die Grünen ein erster Regionaler Wohlfahrtsindex (RWI) für Schleswig-Holstein berechnet." [1]

Diese Aussage vermittelt den Eindruck, die Grüne Landtagsfraktion hätte erstmals dieses Thema aufgegriffen, und dies sei eine offizielle Berechnung durch das Bundesland Schleswig-Holstein. Dies ist jedoch nicht der Fall.

\section{Externe Berechnung des Wohlfahrtsindex}

Vielmehr handelte es sich um ein von der Grünen Landtagsfraktion in Auftrag gegebenes Gutachten unter Mitarbeit der Forschungsstätte der Evangelischen Studiengemeinschaft (FEST), Heidelberg, sowie des Instituts für ökologische Wirtschaftsforschung (IÖW), Berlin. Ein Gutachten in Auftrag zu geben, bedeutet jedoch noch nicht, dass ein ganzes Bundesland seine Berechnungen umstellt.

Diesen Eindruck hat wohl auch Der Spiegel gehabt, der darüber unter der Schlagzeile „Schleswig-Holstein rechnet

sich schön“ berichtete (Teevs 2011). Erst im weiteren Text dieses Spiegel-Artikels erfährt der Leser, dass es sich dabei nicht um die Arbeit eines Bundeslandes, sondern um eine für die Grüne Landtagsfraktion erstellte Studie handelt.

Zudem waren die Grünen auch nicht die Ersten, die das Thema aufgegriffen hatten. Das geschah bereits 20 Jahre früher durch die damals regierende SPD [2]. Diese Initiative basierte auf Erkenntnissen eines am 10. Januar 1990 durchgeführten Forums mit den Referent/ innen: Udo Ernst Simonis und Christian Leipert, Wissenschaftszentrum Berlin, Carsten Stahmer, Statistisches Bundesamt, Wiesbaden, Wilfried Karmaus, Universität Hamburg, Angelika Zahrnt, B.U.N.D. sowie Bernd Struck, Statistisches Landesamt Schleswig-Holstein.

Der Schleswig-Holsteinische Landtag beschloss daraufhin am 21.11. 1991 einstimmig [3], die Landesregierung aufzufordern, dass das Statistische Landesamt in Abstimmung mit dem Statistischen Bundesamt und in enger Zusammenarbeit mit den übrigen Statistischen Landesämtern eine solche Weiterentwicklung der volkswirtschaftlichen Gesamtrechnung vornehmen soll.

\section{Nachhaltige Wirtschaft als Leitbild}

Von den Grünen hat lediglich Frau von Kalben in der Landtagssitzung am 25. 9. 2013 gesagt, dass man auf „eine nachhaltige Wirtschaft als Leitbild“ setze. Und das ist auch gut so. Aber konkrete

\section{Anmerkungen}

[1] Vergleiche dazu das Zitat: „Hinzu kamen ab 2011 Studien in mehreren Bundesländern, zunächst im Auftrag von Landtagsfraktionen der Partei Bündnis '90/Die Grünen, später auch aus Landesregierungen " (Rodenhäuser et al. 2017, S. 32).

[2] Siehe dazu den Landtagsantrag in der Drucksache des Schleswig-Holsteinischen Landtags 12/729 vom 22. Februar 1990.

[3] Siehe Drucksache des Schleswig-Holsteinischen Landtags 12/1708.

[4] Vergleiche Jänicke (1979): Wie das Industriesystem von seinen Mißständen profitiert, Opladen.

[5] Vergleiche Leipert (1989): Die heimlichen Kosten des Fortschritts. Wie Umweltzerstörung das Wirtschaftswachstum fördert, Frankfurt.

\section{Literatur}

Rodenhäuser, D./Held, B./Zieschank, R./Diefenbacher, H. (2018): Orientierung an ewigem Wachstum oder gesellschaftlicher Wohlfahrt? Ökologisches Wirtschaften 33/1: 30-36.

Teevs, C. (2011): Schleswig-Holstein rechnet sich schön. www.spiegel.de/wirtschaft/soziales/ wohlfuehlwachstum-schleswig-holsteinrechnet-sich-schoen-a-763526.html

\section{AUTORIN + KONTAKT}

Dr. Irene Schöne ist Ökologische Ökonomin. Sie war Mitbegründerin des Instituts für Ökologische Wirtschaftsforschung (IÖW) und ist Mitglied im Wissenschaftlichen Beirat. 